\title{
Space-Time Adaptive Processing and Motion Parameter Estimation in Multi-Static Passive Radar Using Sparse Bayesian Learning
}

\author{
Qisong Wu, Member, IEEE, Yimin D. Zhang, Senior Member, IEEE, \\ Moeness G. Amin, Fellow, IEEE, and Braham Himed, Fellow, IEEE
}

\begin{abstract}
Conventional space-time adaptive processing suffers from the requirement of a large number of secondary samples. In this paper, a novel method is proposed to accurately estimate the clutter covariance matrix based on a small number of secondary samples by exploiting the common clutter support across nearby range cells in the angle-Doppler domain. By taking advantage of the intrinsic sparsity of the clutter in the angle-Doppler domain, the recently developed sparse Bayesian learning technique is employed for high-resolution clutter profile estimation. The proposed method does not require the independent and identically distributed secondary sample assumption, and the required number of secondary data samples can be significantly reduced. In addition, we propose a sparse reconstruction based approach to acquire the two-dimensional motion parameters of moving targets by exploiting their group sparsity in the velocity domain in the multi-static passive radar systems. Simulation results verify the effectiveness of the proposed algorithm.
\end{abstract}

Index Terms-Group sparsity, motion parameter estimation, multi-static passive radar, space-time adaptive processing (STAP), sparse Bayesian learning (SBL)

\section{INTRODUCTION}

Passive radar (PR) systems, which utilize broadcast, navigation, and wireless communication signals as sources of opportunity, have recently attracted great interest due to their distinct advantages over conventional active radar systems, primarily in terms of their low cost and covertness [1]. PRs are expected to play an increasing role in future reconnaissance systems [2].

Moving target indication (MTI) and tracking are commonly applied in radar applications aiming at target detection and

Copyright (c) 2015 IEEE. Personal use of this material is permitted. However, permission to use this material for any other purposes must be obtained from the IEEE by sending a request to pubs-permissions@ieee.org.

The work was supported in part by a subcontract with Defense Engineering Corporation for research sponsored by the Air Force Research Laboratory (AFRL) under Contract FA8650-12-D-1376 and by a subcontract with Dynetics, Inc. for research sponsored by the AFRL under Contract FA8650-08D-1303.

Q. Wu was with the Center for Advanced Communications, Villanova University, Villanova, PA 19085, USA. He is now with the Key Laboratory of Underwater Acoustic Signal Processing of Ministry of Education, Southeast University, Nanjing 210096, China (email: qisong.wu@ seu.edu.cn).

Y. D. Zhang was with the Center for Advanced Communications, Villanova University, Villanova, PA 19085, USA. He is now with the Department of Electrical and Computer Engineering, College of Engineering, Temple University, Philadelphia, PA 19122, USA (email: ydzhang@temple.edu).

M. G. Amin is with the Center for Advanced Communications, Villanova University, Villanova, PA 19085, USA.

B. Himed is with the RF Technology Branch, Air Force Research Lab (AFRL/RYMD), WPAFB, OH 45433, USA. motion parameter estimation. The latter has been extensively studied, particularly for active radar platforms, and has benefited from time-frequency analysis [3-5], and motion and range migration compensations [6-8]. However, for airborne radar receivers, those algorithms are effective only when clutter suppression is achieved.

Space-time adaptive processing (STAP) is considered an effective technique to MTI in cluttered environments. STAP, in essence, performs signal processing in the joint angle-Doppler domain to better separate moving targets from clutter $[9,10]$. Application of STAP techniques requires a large number of secondary data samples from other range gates to estimate the clutter covariance matrix (CCM) [9]. More specifically, for a STAP system consisting of $N$ antennas and $L$ azimuth samples, the well-known Reed-Mallett-Brennan's (RMB) rule states that $2 N L$ independent and identically distributed (i.i.d.) secondary data samples are required to achieve an output SINR that is within $3 \mathrm{~dB}$ loss from the Clairvoyant solution [11]. Although a number of reduced-rank STAP algorithms have been developed to robustly estimate the CCM with a smaller number of secondary data samples (e.g., [10, 12-14]), the required number of secondary data samples is still at least twice the rank of the dominant clutter subspace for a satisfactory performance. By jointly exploiting the reduced-rank technique and the structure of the covariance matrix $[15,16]$, this number can be further reduced. However, this approach is still considered impractical in passive radar systems due to the narrow signal bandwidth and the corresponding low range resolution. For example, a Digital Video BroadcastingTerrestrial (DVB-T) channel occupies a 7.6 MHz bandwidth in the $450-900 \mathrm{MHz}$ band and its corresponding bistatic range resolution is approximately $20 \mathrm{~m}$. The range resolution becomes even coarser when signals with a narrower bandwidth, such as the Digital Audio Broadcasting (DAB) broadcast and Global System for Mobile Communications (GSM) signals, are used. With a much lower range resolution as compared to conventional active radar systems, it is often difficult to acquire a sufficient number of i.i.d. secondary data samples required for the estimation of a full-rank CCM [17, 18].

In order to relax the condition on the number of secondary samples, a more recent approach is to exploit the latest advances of sparsity reconstruction and compressive sensing (CS) techniques. By taking advantage of the intrinsic sparsity of the clutter in the angle-Doppler domain, the sparse clutter spectrum can be recovered by using only one or a few samples 
[19-23]. In essence, we formulate the sparse clutter profile estimation as a regularized optimization problem, and then construct the CCM based on the estimated clutter profiles to achieve effective clutter suppression. In [19], clutter suppression is achieved by assuming the knowledge of the support of the clutter ridge in the angle-Doppler plane. By applying a mask to the assumed clutter ridge, moving targets are estimated as the sparse solution in the angle-Doppler domain outside this clutter ridge. In [20], the clutter at each secondary range cell is separately estimated in the Bayesian framework. The estimated clutter at each range cell is used to compute the CCM and perform STAP in the traditional sense. In [21, 22], two approaches are proposed for the clutter profile estimation by utilizing multiple data samples where the clutter profile is estimated separately in each range cell. The former averages the estimated clutter profiles to construct the CCM, whereas in the latter method, the maximum value is chosen for each angleDoppler entry from all range cells being evaluated. In [23], the STAP processor is assumed to be persymmetry in both spatial and azimuthal domains, and a complex sparse clutter estimation problem is cast into a group sparsity formulation to be solved in the multi-task Bayesian CS framework. We maintain that none of the above methods utilizes the group sparsity of the clutter across range cells for improved clutter support or profile estimation. Except [19] in which the support of the clutter ridge in the angle-Doppler plane is assumed to be known, the other aforementioned algorithms assume that the moving target signals in the range cell under test are excluded in the reconstructed clutter profiles. While this assumption may hold true for weak target signals due to the non-linear behavior of sparse reconstruction, it is nevertheless difficult to guarantee such an outcome in various situations.

A separate issue arising in the sparse reconstruction of the clutter profile is its angle-Doppler domain resolution. In addition to satisfying the sparsity requirement, most existing CS algorithms, such as greedy algorithms like the orthogonal matching pursuit (OMP) [24] and regularized optimization algorithms like the LASSO [25], generally expect a low coherence measurement matrix. A much higher number of measurement samples would be required when the measurement matrix is highly coherent [26-28]. On the other hand, sparse Bayesian learning (SBL) methods, which are generally developed under the relevance vector machine (RVM) framework [29], form a different class of effective tools for sparse signal reconstruction. These methods are known to achieve improved performance in many applications, particularly when the measurement matrix is of a high coherence [30]. Highresolution sparse reconstruction problems are encountered in the underlying STAP $[22,23]$ as well as a large class of radar applications, such as in direction-of-arrival (DOA) estimation [31, 32], microwave imaging [33-35], and synthesis of linear array $[36,37]$. While the original SBL framework is developed to handle real-valued data, a number of methods have been made available to process complex-valued data in the context of the SBL framework [34, 38]. In [34], the complex-valued problem is reformulated as fictitious real-valued tasks with interrelationship between the real and imaginary components. The idea that common parameters are shared by the real and imaginary components is taken into account in [38]. In this paper, we use the SBL method developed in the context of complex Gaussian distribution [39] to estimate the complex-valued clutter profiles. This method directly models the complex-valued sparse reconstruction problem in the context of complex Gaussian distributions, and the problem is solved using the standard RVM approach [29].

In this paper, we propose a novel approach to accurately estimate the CCM and implement STAP based on a small number of secondary data samples. By exploiting the group sparsity that nearby range cells share the same non-zero clutter support in the angle-Doppler domain, we first use the secondary data samples to estimate the common non-zero clutter support that excludes the target signals. The estimated clutter support is then used to estimate the clutter profile in the range cell under test with the target signals excluded. The proposed method does not require the i.i.d. secondary sample assumption, and the number of secondary data samples can be significantly reduced. The SBL algorithm is introduced to improve clutter profile reconstruction, particularly when the measurement matrix has a high coherence.

A number of methods have been developed to consider the group sparse problems and multi-channel observations. In the block orthogonal matching pursuit (BOMP) [40] and group Lasso (GLasso) [41], the group sparsity is considered by evaluating the $l_{2}$-norm of the member entries in each group. The multi-task CS algorithm [42] is a sparse Bayesian learning method where the group sparsity is accounted using the shared priors. The M-FOCUSS algorithm [43] is another popularly used group sparse reconstruction method that is developed under the multiple measurement vector (MMV) model. There are different approaches that deal with multichannel observations. Reference [44] considers the joint sparse signal model to account for multiple aspect observations in a wide-angle synthetic aperture radar (SAR) imaging. The sparsity similarity between different aspect angles is considered through the change in the scattering magnitude at each pixel. A similar technique is applied in [45] to achieve enhanced image from multichannel complex-valued images while preserving the cross-channel information.

In this paper, considering the fact that the clutter profiles share the same non-zero clutter support across nearby range cells, we incorporate group sparsity into sparse Bayesian learning. This incorporation is generally performed within the multi-task CS framework [42]. In this case, the group sparsity is encouraged by exploiting a common precision parameter for each discretized clutter entry corresponding to all range cells. We extend the multi-task CS algorithm, which is developed under real-valued data model, to process complex-valued variables by using complex Gaussian distributions [39]. It is important to note that the observation model is characterized by the MMV model which assumes identical measurement matrices for observations from nearby range cells in each bisatic pair. It is shown that, while only an approximate solution of the aforementioned precision parameter can be obtained in the general multi-task model, we are able to derive an analytical solution of these parameters under the MMV model. 
Another important task of a radar STAP system is the target detection and motion parameter estimation of moving targets. Unlike conventional mono-static radars, which only estimate the radial velocity as the result of single-direction Doppler frequency, the multi-static operation of a passive radar system enables two-dimensional (2-D) motion parameter estimation $[46,47]$. In addition, multiple moving targets may be present in a region and are inseparable in range due to the low range resolution, rendering motion parameter estimation difficult. The task of target detection and motion parameter estimation becomes possible only after effective suppression of the clutter. In this paper, we propose a novel sparse reconstruction based approach to acquire 2-D motion parameters by exploiting the group sparsity in the velocity domain. The proposed approach enables 2-D velocity estimation of each moving target and outperforms other techniques that non-coherently combine multi-static STAP outputs.

To summarize, the key contribution of the paper is twofold: (1) We propose a novel approach for reliable and high-resolution CCM estimation based on a small number of secondary samples by using the group SBL approach to exploit the group sparsity of clutter profiles across the nearby range cells. There is no requirement of i.i.d. property of the secondary samples. (2) We obtain the 2-D motion parameters of multiple moving targets by utilizing the group sparsity in the velocity domain and coherently combining multi-static measurements.

Notations: We use lower-case (upper-case) bold characters to denote vectors (matrices). In particular, $\mathbf{I}_{N}$ denotes the $N \times$ $N$ identity matrix. (.)* represents complex conjugate, whereas $(.)^{T}$ and $(.)^{H}$, respectively, denote the transpose and conjugate transpose of a matrix or vector. $\operatorname{diag}(\mathbf{x})$ represents a diagonal matrix that uses the elements of $\mathbf{x}$ as its diagonal elements , and $\operatorname{std}(x)$ denotes the standard deviation of $x .\|\cdot\|_{2}^{2}$ implies the Euclidean $\left(l_{2}\right)$ norm of a vector, whereas $\|\cdot\|_{1}$ denotes the $l_{1}$ norm. $\mathrm{P}_{\mathrm{r}}(\cdot)$ expresses the probability density function (pdf), and $\mathcal{C N}(x \mid a, b)$ denotes the random variable $x$ follows a complex Gaussian distribution with mean $a$ and variance $b$. In addition, $\otimes$ is the Kronecker product, and $\operatorname{vec}(\cdot)$ is the vectorization operator.

\section{SYSTEM MODEL}

\section{A. Radar Geometry and Signal Model}

Consider a multi-static radar scene consisting of $K$ stationary transmitters and a moving receiver as depicted in Fig. 1. The $i$ th transmitter is assumed to be located at a known position $\mathbf{p}_{\mathrm{T} i}=\left[p_{\mathrm{T} i, x}, p_{\mathrm{T} i, y}, p_{\mathrm{T} i, z}\right]^{T}$ and uses a single antenna to emit a signal at frequency $f_{i}, i=1, \ldots, K$. The radar receiver, which utilizes an $N$-element uniform linear array (ULA) with inter-element spacing $d$, moves in a straight line with a constant horizontal velocity $v_{R}$.

For convenience of notation and without loss of generality, we assume that the receiver moves in the $x$-direction. The initial position of the receiver is expressed in the Cartesian coordinate system as $\mathbf{p}_{\mathrm{R}}(0)=\left[p_{\mathrm{R}, x}(0), 0, H_{\mathrm{R}}\right]^{T}$, and the velocity vector is $\mathbf{v}_{R}=\left[v_{R}, 0,0\right]^{T}$. As such, the position of

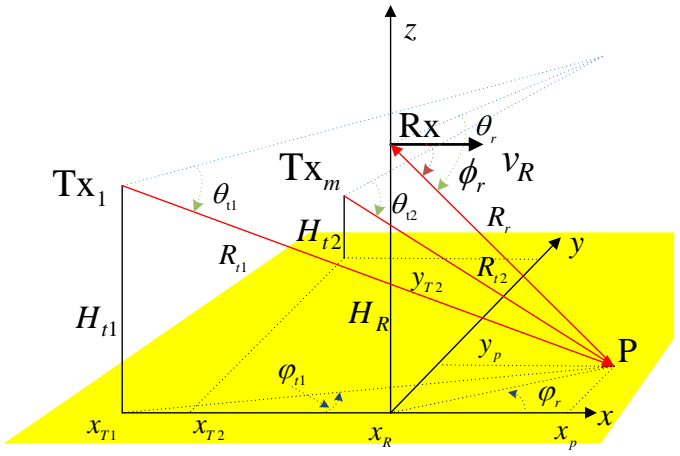

Fig. 1. Multi-static passive radar geometry.

the receiver at time instant $t$ is expressed as

$$
\mathbf{p}_{\mathrm{R}}(t)=\left[p_{\mathrm{R}, x}(0)+v_{\mathrm{R}} t, 0, H_{\mathrm{R}}\right]^{T} .
$$

The signal vector received at the receive array is the superposition of the signals corresponding to the $K$ transmitters, expressed as,

$$
\mathbf{x}(t)=\sum_{k=1}^{K} \mathbf{x}_{k}(t)+\mathbf{n}(t)=\sum_{k=1}^{K} \mathbf{x}_{k s}(t)+\sum_{k=1}^{K} \mathbf{x}_{k c}(t)+\mathbf{n}(t),
$$

where $\mathbf{x}_{k}(t)$ is the combined received signal corresponding to the $k$ th illuminated source. It consists of two components: the target signal $\mathbf{x}_{k s}(t)$ and the clutter $\mathbf{x}_{k c}(t)$. In addition, $\mathbf{n}(t)$ is the additive noise, which is characterized as i.i.d. complex Gaussian with zero mean. The target signal and the clutter will be described in the next two subsections.

Note that, in the above expression, we have assumed that the received signal is properly conditioned. That is, the directpath signal from the transmitters to the receiver is perfectly suppressed through, for example, spatial filtering utilizing the known geometric relationship between each illuminator and the receiver. In addition, we assume that the signal waveform transmitted from each illuminator is perfectly reconstructed as the result of channel error correction coding. These are standard assumptions commonly used for passive radar [46, 48].

\section{B. Target Signal}

Assume that $Q$ ground moving targets are located in close proximity in bistatic ranges, rendering them unresolvable. The elevation coordinate of all targets is assumed to be 0 . The $q$ th ground moving target is located at $\mathbf{p}_{q}=\left[x_{q}, y_{q}, 0\right]^{T}$ with a velocity of $\mathbf{v}_{q}=\left[v_{x q}, v_{y q}, 0\right]^{T}$. The desired signal $\mathbf{x}_{s}(t)$ received at the receiver is expressed as

$$
\begin{aligned}
\mathbf{x}_{s}(t) & =\sum_{k=1}^{K} \mathbf{x}_{k s}(t) \\
& =\sum_{k=1}^{K} \sum_{\substack{q=1\\
}}^{Q} \frac{\sqrt{P_{\mathrm{T}_{k}}} G_{k q} \sigma_{k q}}{r_{\mathrm{T}_{k} q} r_{q \mathrm{R}}} s_{k}\left[t-\tau_{\mathrm{T}_{k} q}(t)-\tau_{q \mathrm{R}}(t)\right] \\
& \times e^{-j 2 \pi f_{k}\left[\tau_{\mathrm{T}_{k} q}(t)+\tau_{q \mathrm{R}}(t)\right]} \mathbf{a}_{k}\left(\phi_{q}\right),
\end{aligned}
$$

where $P_{\mathrm{T}_{k}}$ is the transmit signal power of the $k$ th illuminator, $G_{k q}$ represents the antenna gain of the $k$ th illuminator in the 
direction of the $q$ th target, and $\sigma_{k q}$ is a complex reflection coefficient associated with the radar cross section (RCS) of the $q$ th target and depends on the specific illuminator $k$ because the RCS varies with the aspect angle. In addition, $s_{k}(t)$ is the waveform transmitted from the $k$ th illuminator, $\tau_{\mathrm{T}_{k} q}(t)=r_{\mathrm{T}_{k} q}(t) / c$ and $\tau_{q \mathrm{R}}(t)=r_{q \mathrm{R}}(t) / c$ are the time delays respectively corresponding to the range between the $k$ th illuminator and the $q$ th moving target,

$$
r_{\mathrm{T}_{k} q}(t)=\left\|\mathbf{p}_{\mathrm{T}_{k}}-\mathbf{p}_{q}(t)\right\|
$$

and the range between the $q$ th moving target and the receiver,

$$
r_{q \mathrm{R}}(t)=\left\|\mathbf{p}_{q}(t)-\mathbf{p}_{R}(t)\right\|,
$$

where $c$ is the velocity of light. Furthermore,

$$
\mathbf{a}_{k}\left(\phi_{q}\right)=\left[1, e^{j \kappa_{k} d \sin \left(\phi_{q}\right)}, \ldots, e^{j \kappa_{k}(N-1) d \sin \left(\phi_{q}\right)}\right]^{T} \in \mathcal{C}^{N}
$$

is the steering vector of the receive array toward the direction of the target with a DOA $\phi_{q}$, which is defined as the cone angle of the $q$ th target with respect to the $x$-axis. In the above expression, $\kappa_{k}=2 \pi / \lambda_{k}$ is the wavenumber with $\lambda_{k}=c / f_{k}$ denoting the wavelength of the signal transmitted from the $k$ th illuminator. Note that, the DOA is assumed to be time-invariant in the sense that its change during the coherent processing interval (CPI) is negligible.

For a given area, specified by delay $\tau_{i, n}$ for the $i$ th bistatic pair, we perform matched filtering by using the reconstructed waveform of the signal emitted from the $i$ th illuminator as the reference signal. The matched filter output at the $L$ azimuth time instants $t_{l}=l T, l=0, \ldots, L-1$, is given by

$$
\begin{aligned}
\mathbf{y}_{i s}^{(n)}\left(t_{l}\right) & =\int_{t_{l}}^{t_{l}+T} \mathbf{x}_{s}(t) s_{i}^{*}\left(t-\tau_{i, n}\right) e^{j 2 \pi f_{i} \tau_{i, n}} d t \\
& =\sum_{q=1}^{Q} \frac{\sqrt{P_{\mathrm{T}_{i}}} G_{i q} \sigma_{i q}}{r_{\mathrm{T}_{i}} r_{q \mathrm{R}}} \mathbf{a}_{i}\left(\phi_{q}\right) \int_{t_{l}}^{t_{l}+T} s_{i}\left[t-\tau_{\mathrm{T}_{i} q}(t)-\tau_{q \mathrm{R}}(t)\right] \\
& \times s_{i}^{*}\left(t-\tau_{i, n}\right) e^{-j 2 \pi f_{i}\left[\tau_{\mathrm{T}_{i} q}(t)+\tau_{q \mathrm{R}}(t)-\tau_{i, n}\right]} d t,
\end{aligned}
$$

where $r_{\mathrm{T}_{i} q}$ and $r_{q \mathrm{R}}$ in the denominator are assumed to be constant by neglecting the effect of variation of $r_{\mathrm{T}_{i} q}$ and $r_{q \mathrm{R}}$ on the amplitude within the short CPI. The small phase change within the matched filtering window [48] permits choosing the delay $\tau_{i, n}$ to align the received signal with the reference signal during the center of the CPI, i.e., $\tau_{i, n}=\left(\tau_{\mathrm{T}_{i} n}+\tau_{n \mathrm{R}}\right)+(L-$ 1) $T / 2$. By ignoring the range migration issue due to the short CPI, the above expression is simplified as

$$
\mathbf{y}_{i s}^{(n)}\left(t_{l}\right)=\sum_{q=1}^{Q} \frac{\sqrt{P_{\mathrm{T}_{i}}} G_{i q} \sigma_{i q}}{r_{\mathrm{T}_{i} q} r_{q \mathrm{R}}} \rho_{i} \mathbf{a}_{i}\left(\phi_{q}\right) e^{j 2 \pi \nu_{i q}\left[t_{l}-(L-1) T / 2\right]},
$$

where $\rho_{i}=\rho_{i}(0)$, with

$$
\rho_{i}(\tau)=\int_{t_{l}}^{t_{l}+T} s_{i}(t) s_{i}^{*}(t+\tau) d t
$$

denoting the signal auto-correlation function, which is independent of $t_{l}$ as most digital waveforms have a stable autocorrelation property [2]. In addition, $\nu_{i q}$ denotes the Doppler frequency of the $q$ th moving target which is determined by the change rate of the combined bistatic range, expressed as

$$
\begin{aligned}
\nu_{i q}= & -\frac{1}{\lambda_{i}} \cdot \frac{d}{d t}\left[r_{\mathrm{T}_{i} q}(t)+r_{q \mathrm{R}}(t)\right] \\
= & -\frac{1}{\lambda_{i}} \cdot\left[\frac{\left[\mathbf{p}_{\mathrm{T}_{i}}-\mathbf{p}_{q}(0)\right]^{T} \cdot \mathbf{v}_{q}}{\left\|\mathbf{p}_{\mathrm{T}_{i}}-\mathbf{p}_{q}(0)\right\|}+\frac{\left[\mathbf{p}_{\mathrm{R}}(0)-\mathbf{p}_{q}(0)\right]^{T} \cdot \mathbf{v}_{q}}{\left\|\mathbf{p}_{\mathrm{R}}(0)-\mathbf{p}_{q}(0)\right\|}\right. \\
& \left.+\frac{\left[\mathbf{p}_{\mathrm{R}}(0)-\mathbf{p}_{q}(0)\right]^{T} \cdot \mathbf{v}_{R}}{\left\|\mathbf{p}_{\mathrm{R}}(0)-\mathbf{p}_{q}(0)\right\|}\right],
\end{aligned}
$$

where $\mathbf{p}_{q}(0)$ is the the initial position of the $q$ th moving target. The total Doppler frequency $\nu_{i q}$ can be regarded as the combination of two components. The first one, which corresponds to the first two terms in Eq. (10), represents the contribution of moving target, whereas the second component, given as the third term in Eq. (10), represents the contribution of the moving receiver. Note that the first component disappears for stationary clutter, which is discussed in the following subsection.

Conventional mono-static STAP systems detect moving targets and estimate the target Doppler frequencies projected onto the observation direction, thus failing to acquire the 2-D motion parameters. In the multi-static operation, on the other hand, 2-D motion parameters can be estimated due to different transmitting aspect angles. It is clear from Eq. (10) that, while the second and third terms are common to all transmitters, the first term varies with the illuminator positions due to the diverse observation aspect angles. These illuminator-dependent Doppler frequencies provide sufficient information for the receiver to acquire 2-D motion parameters of moving targets. Motion parameter estimation is discussed in Section V.

Stack $\mathbf{y}_{i s}^{(n)}\left(t_{l}\right)$ over the $L$ collected azimuth time samples as

$$
\begin{aligned}
\overline{\mathbf{y}}_{i s} & =\left[\left[\mathbf{y}_{i s}^{(n)}\left(t_{0}\right)\right]^{T},\left[\mathbf{y}_{i s}^{(n)}\left(t_{1}\right)\right]^{T}, \ldots,\left[\mathbf{y}_{i s}^{(n)}\left(t_{L-1}\right)\right]^{T}\right]^{T} \\
& =\sum_{q=1}^{Q} \frac{\sqrt{P_{\mathrm{T}_{i}}} G_{i q} \sigma_{i q}}{r_{\mathrm{T}_{i}} r_{q} \mathrm{R}} \rho_{i} \mathbf{h}\left(\nu_{i q}, \phi_{q}\right)
\end{aligned}
$$

where

$$
\mathbf{h}\left(\nu_{i q}, \phi_{q}\right)=\mathbf{b}\left(\nu_{i q}\right) \otimes \mathbf{a}\left(\phi_{q}\right) \in \mathcal{C}^{N L}
$$

is the spatio-temporal signature of the $q$ th target, and

$$
\begin{gathered}
\mathbf{b}\left(\nu_{i q}\right)=\left[e^{-j 2 \pi \nu_{i q}(L-1) T / 2}, e^{-j 2 \pi \nu_{i q}(L-3) T / 2}, \ldots,\right. \\
\left.e^{j 2 \pi \nu_{i q}(L-1) T / 2}\right]^{T} \in \mathcal{C}^{L}
\end{gathered}
$$

is the temporal signature vector of the same target.

\section{Clutter}

The clutter received at the receiver is considered as a summation of $N_{c}$ statistically independent scatterers, expressed as,

$$
\begin{aligned}
\mathbf{x}_{c}(t)= & \sum_{k=1}^{K} \mathbf{x}_{k c}(t) \\
= & \sum_{k=1}^{K} \sum_{m=1}^{N_{c}} \frac{\sqrt{P_{\mathrm{T}_{k}}} G_{k m} \sigma_{k m}}{r_{\mathrm{T}_{k} m} r_{m \mathrm{R}}(t)} s_{k}\left[t-\tau_{\mathrm{T}_{k} m}(t)-\tau_{m \mathrm{R}}(t)\right] \\
& \times e^{-j 2 \pi f_{k}\left(\tau_{\mathrm{T}_{k} m}+\tau_{m \mathrm{R}}(t)\right)} \mathbf{a}_{k}\left(\phi_{m}\right),
\end{aligned}
$$


where, similar to the target signal, $r_{\mathrm{T}_{k} m}$ is the range from the $k$ th illuminator and the $m$ th clutter scatterer with $m \in$ $1, \cdots, N_{c}, r_{m \mathrm{R}}(t)$ is the range from the same scatterer to the receiver, $\tau_{\mathrm{T}_{k} m}$ and $\tau_{m \mathrm{R}}(t)$ are respectively the delays corresponding to $r_{\mathrm{T}_{k} m}$ and $r_{m \mathrm{R}}(t)$. In addition, $\sigma_{k m}$ is the reflectivity of the $m$ th clutter scatterer with respect to the $k$ th illuminator, and $\mathbf{a}_{k}\left(\phi_{m}\right)$ is its corresponding steering vector observed at the receive array. Similar to processing the target signals, the matched filter output of the clutter signals at azimuth time $t_{l}=l T, l=0, \ldots, L-1$, corresponding to the $i$ th bistatic pair and the $n$th range bin, is expressed as

$\mathbf{y}_{i c}^{(n)}\left(t_{l}\right)=\sum_{m=1}^{N_{c}} \frac{\sqrt{P_{\mathrm{T}_{i}}} G_{i m} \sigma_{i m}}{r_{\mathrm{T}_{i} m} r_{m \mathrm{R}}\left(t_{l}\right)} \rho_{i} \mathbf{a}_{i}\left(\phi_{m}\right) e^{j 2 \pi \nu_{i m}\left(t_{l}-(L-1) T / 2\right)}$

where

$$
\nu_{i m}=-\frac{1}{\lambda_{i}} \frac{d}{d t} r_{m \mathrm{R}}(t)=-\frac{1}{\lambda_{i}} v_{\mathrm{R}} \cos \phi_{m}
$$

is the Doppler frequency of the $m$ th clutter scatterer and is determined by the scatterer-receiver path, where $\phi_{m}$ is the cone angle between the direction of the scatterer and the array baseline. It should be noted in Eq. (16) that the clutter Doppler frequency depends on the receiver velocity and the transmit frequency, but is independent of range cells. This characteristic provides a feasible way to acquire the clutter profiles in the angle-Doppler domain by exploiting the group sparsity across the nearby range cells.

Stacking $\mathbf{y}_{i c}^{(n)}\left(t_{l}\right)$ over the $L$ collected azimuth time samples yields

$$
\begin{aligned}
\overline{\mathbf{y}}_{i c} & =\left[\left[\mathbf{y}_{i c}^{(n)}\left(t_{0}\right)\right]^{T},\left[\mathbf{y}_{i c}^{(n)}\left(t_{1}\right)\right]^{T}, \ldots,\left[\mathbf{y}_{i c}^{(n)}\left(t_{L-1}\right)\right]^{T}\right]^{T} \\
& =\sum_{m=1}^{N_{c}} \frac{\sqrt{P_{\mathrm{T}_{i}}} G_{i m} \sigma_{i m}}{r_{\mathrm{T}_{i} m} r_{m \mathrm{R}}\left(t_{l}\right)} \rho_{i} \mathbf{h}\left(\nu_{i m}, \phi_{m}\right)
\end{aligned}
$$

where

$$
\mathbf{h}\left(\nu_{i m}, \phi_{m}\right)=\mathbf{b}\left(\nu_{i m}\right) \otimes \mathbf{a}_{i}\left(\phi_{m}\right) \in \mathcal{C}^{N L}
$$

is the spatio-temporal signature of the $m$ th scatterer, and

$$
\begin{gathered}
\mathbf{b}\left(\nu_{i m}\right)=\left[e^{-j 2 \pi \nu_{i m}(L-1) T / 2}, e^{-j 2 \pi \nu_{i m}(L-3) T / 2}, \ldots,\right. \\
\left.e^{j 2 \pi \nu_{i m}(L-1) T / 2}\right]^{T} \in \mathcal{C}^{L}
\end{gathered}
$$

is the temporal signature vector of the $m$ th clutter scatterer.

\section{Group Sparse BAyEsian LEARning Method}

Because of the group sparsity of the clutter profile in the angle-Doppler domain across nearby range cells, group sparse reconstruction approaches can be applied and are considered effective for clutter profile estimation. In this paper, we use the group SBL method developed in the context of complex Gaussian distribution [39] to reconstruct the sparse clutter profile. This approach is briefly summarized below.

We discretize the angle-Doppler domain into a grid with $N_{d}$ Doppler samples and $N_{s}$ azimuth samples. Denote $\mathbf{w}_{k}$ as an $M \times 1$ vector that includes vectorized clutter entries defined in the entire angle-Doppler domain, where $M=N_{d} N_{s}$. Consider a general multi-task model where the complex measurement vector of spatio-temporal samples in the $k$ th bistatic pair is described as

$$
\mathbf{y}_{k}=\mathbf{\Psi}_{k} \mathbf{w}_{k}+\varepsilon_{k}, \quad k \in[1, \cdots, K],
$$

where the dimension of vector $\mathbf{y}_{k}$ is $N_{0}=N L, \mathbf{\Psi}_{k} \in \mathcal{C}^{N_{0} \times M}$ is the $k$ th measurement matrix, and $\varepsilon_{k}$ denotes an additive complex Gaussian noise. When $K=1$, it is a canonical sparse reconstruction problem for the coefficient vector. The likelihood of the observed data $\mathbf{y}_{k}$ can be expressed as,

$$
\begin{aligned}
\operatorname{Pr}\left(\mathbf{y}_{k} \mid \mathbf{w}_{k}, \mathbf{\Psi}_{k}, \beta_{0}\right) & =\mathcal{C N}\left(\mathbf{y}_{k} \mid \mathbf{\Psi}_{k} \mathbf{w}_{k}, \beta_{0} \mathbf{I}_{N}\right) \\
& =\frac{1}{\left(\pi \beta_{0}\right)^{N_{0}}} \exp \left(-\frac{1}{\beta_{0}}\left\|\mathbf{y}_{k}-\mathbf{\Psi}_{k} \mathbf{w}_{k}\right\|_{2}^{2}\right),
\end{aligned}
$$

where $\beta_{0}$ is the variance of the complex Gaussian noise. The prior distribution of the weight vector $\mathbf{w}_{m}$. = $\left[w_{m 1}, \cdots, w_{m K}\right]$, which is the $m$ th row of $\mathbf{W}=$ $\left[\mathbf{w}_{1}, \cdots, \mathbf{w}_{K}\right] \in \mathcal{C}^{M \times K}$, is modeled as a zero-mean complex Gaussian distributions with unknown precision (reciprocal of variance) $\alpha_{m}$, i.e.,

$$
\mathbf{w}_{m} \cdot \sim \mathcal{C N}\left(\mathbf{w}_{m \cdot} \mid \mathbf{0}, \alpha_{m}^{-1} \mathbf{I}_{K}\right)
$$

where $\left\{\alpha_{m}\right\}_{m=1}^{M}$ is a nonnegative parameter controlling the group sparsity of $\mathbf{w}_{m}$. When $\alpha_{m}=\infty$, the $m$ th row $\mathbf{w}_{m}$. becomes zero. During the learning procedure, most elements of $\alpha_{m}$ tend to be infinite, due to the mechanism of automatic relevance determination [29]. Thus sparsity at the group level is encouraged.

In the group SBL framework, the posterior distribution for $\mathbf{w}_{k}$ can be evaluated analytically based on the Bayes' rule as

$$
\operatorname{Pr}\left(\mathbf{w}_{k} \mid \mathbf{y}_{k}, \boldsymbol{\Psi}_{k}, \boldsymbol{\alpha}, \beta_{0}\right)=\mathcal{C N}\left(\mathbf{w}_{k} \mid \boldsymbol{\mu}_{k}, \boldsymbol{\Sigma}_{k}\right),
$$

where

$$
\begin{aligned}
& \boldsymbol{\mu}_{k}=\beta_{0}^{-1} \boldsymbol{\Sigma}_{k} \boldsymbol{\Psi}_{k}^{H} \mathbf{y}_{k}, \quad k \in[1, \cdots, K], \\
& \boldsymbol{\Sigma}_{k}=\left(\beta_{0}^{-1} \boldsymbol{\Psi}_{k}^{H} \boldsymbol{\Psi}_{k}+\mathbf{A}\right)^{-1},
\end{aligned}
$$

where $\mathbf{A}=\operatorname{diag}\left(\alpha_{1}, \cdots, \alpha_{M}\right)$. Once the parameters $\boldsymbol{\alpha}$ and $\beta_{0}$ are estimated, the maximum a posteriori (MAP) estimate of $\mathbf{w}_{k}$, denoted by $\hat{\mathbf{w}}_{k}$, can be obtained from the posterior mean as,

$$
\hat{\mathbf{w}}_{k}=\boldsymbol{\mu}_{k}, \quad k \in[1, \cdots, K] .
$$

With known $\boldsymbol{\alpha}$ and $\beta_{0}$, the mean and covariance of each scattering coefficient are derived in (23) and (24). The associated learning problem, in the context of the RVM, thus becomes searching for the parameters $\boldsymbol{\alpha}$ and $\beta_{0}$. The empirical Bayesian estimate for $\boldsymbol{\alpha}$ is determined by maximizing the marginal likelihood [29, 49], or equivalently, its logarithm

$$
\left\{\boldsymbol{\alpha}, \beta_{0}\right\}=\underset{\boldsymbol{\alpha}, \beta_{0}}{\operatorname{argmax}} \mathcal{L}\left(\boldsymbol{\alpha}, \beta_{0}\right),
$$


where

$$
\begin{aligned}
\mathcal{L}\left(\boldsymbol{\alpha}, \beta_{0}\right) & =\sum_{k=1}^{K} \log \mathrm{P}_{\mathrm{r}}\left(\mathbf{y}_{k} \mid \boldsymbol{\alpha}, \beta_{0}\right) \\
& \equiv \sum_{k=1}^{K}-\log \left|\mathbf{C}_{k}\right|-\mathbf{y}_{k}^{H} \mathbf{C}_{k}^{-1} \mathbf{y}_{k},
\end{aligned}
$$

in which $\mathbf{C}_{k}=\beta_{0} \mathbf{I}+\boldsymbol{\Psi}_{k} \mathbf{A}^{-1} \boldsymbol{\Psi}_{k}^{H}$. Following the fast greedy algorithm based on the marginal likelihood maximization criterion [50], we acquire the approximate solution of $\alpha_{m}$ in the multi-task model as [42]

$$
\alpha_{m}^{\text {(new) }}\left\{\begin{array}{l}
\approx \frac{K}{\sum_{k=1}^{K} \frac{1}{a_{k, m}^{2}}\left(b_{k, m}^{2}-a_{k, m}\right)}, \\
=\infty, \quad \text { if } \sum_{k=1}^{K} \frac{1}{a_{k, m}^{2}}\left(b_{k, m}^{2}-a_{k, m}\right)>0, \\
\text { if } \sum_{k=1}^{K} \frac{1}{a_{k, m}^{2}}\left(b_{k, m}^{2}-a_{k, m}\right) \leq 0,
\end{array}\right.
$$

where $a_{k, m} \triangleq \boldsymbol{\psi}_{k, m}^{H} \mathbf{C}_{k,-m}^{-1} \boldsymbol{\psi}_{k, m}, b_{k, m} \triangleq \boldsymbol{\psi}_{k, m}^{H} \mathbf{C}_{k,-m}^{-1} \mathbf{y}_{k}$, $\boldsymbol{\psi}_{k, m}$ represents the $m$ th column in $\boldsymbol{\Psi}_{k}$, and $\mathbf{C}_{k,-m}$ is $\mathbf{C}_{k}$ with the contribution of atom $\boldsymbol{\psi}_{k, m}$ removed.

When $\boldsymbol{\Psi}_{1}=\cdots=\boldsymbol{\Psi}_{K}=\boldsymbol{\Psi}$, the model in Eq. (20) reduces to a typical multiple measurement vector (MMV) model [43]. In this case, unlike approximate solutions in the multi-task model, we can derive an analytical solution of $\alpha_{m}$ as,

$$
\alpha_{m}^{\text {(new) }}=\left\{\begin{array}{cl}
\frac{K a_{m}^{2}}{\sum_{k=1}^{K} b_{k, m}^{2}-K a_{m}}, & \text { if } \sum_{k=1}^{K} b_{k, m}^{2}>K a_{m}, \\
\infty, & \text { if } \sum_{k=1}^{K} b_{k, m}^{2} \leq K a_{m},
\end{array}\right.
$$

where $a_{m} \triangleq \boldsymbol{\psi}_{m}^{H} \mathbf{C}_{-m}^{-1} \boldsymbol{\psi}_{m}, \boldsymbol{\psi}_{m}$ represents the $m$ th column in the common measurement matrix $\boldsymbol{\Psi}$, and $\mathbf{C}_{-m}$ is $\mathbf{C}=$ $\beta_{0} \mathbf{I}+\boldsymbol{\Psi} \mathbf{A}^{-1} \Psi^{H}$ with the contribution of atom $\boldsymbol{\psi}_{m}$ removed. It is observed that these two solutions of $\alpha_{m}^{\text {(new) }}$ are consistent.

Following the approach of MacKay [49], we differentiate Eq. (26) with respect to $\beta_{0}$ and set the result to zero, yielding

$$
\beta_{0}^{(\text {new })}=\frac{\sum_{k=1}^{K}\left\|\mathbf{y}_{k}-\mathbf{\Psi}_{k} \boldsymbol{\mu}_{k}\right\|_{2}^{2}}{\sum_{k=1}^{K}\left[N_{0}-M+\sum_{m=1}^{M} \alpha_{m} \Sigma_{k,(m m)}\right]}
$$

where $\Sigma_{k,(m m)}$ denotes the $(m, m)$ th entry in the matrix $\boldsymbol{\Sigma}_{k}$.

Instead of going through all the elements in the $M$ groups in each iteration, the group SBL algorithm described above operates in a constructive manner, i.e., sequentially adds (or deletes) candidate atom to the model until all relevant atoms, for which the associate weights are non-zero, have been included. Therefore, the computational complexity of the algorithm is $\mathcal{O}\left(K M m_{0}^{2}\right)$, with $m_{0}$ denoting the sparsity, which is on the same order as the multi-task CS [42]. The complexity is linearly proportional to $M$ and thus is comparable to that of the well-known M-FOCUSS [43].

\section{Clutter Profile Estimation and Suppression}

\section{A. Group-Sparsity Representation of Clutter Profile across Range Cells}

Because the non-zero support of clutter profiles is shared across nearby range cells, we first employ a small number of secondary samples to learn the common clutter support. The exact clutter profile in the range cell under test is then obtained through the SBL method by only considering the clutter entries within the learned clutter support.

According to the signal and clutter model described in Section II, the output of the matched filter of the received signal in the range cell under test for the $i$ th bistatic pair is expressed as

$$
\overline{\mathbf{y}}_{i}^{(\mathrm{t})}=\overline{\mathbf{y}}_{i c}^{(\mathrm{t})}+\overline{\mathbf{y}}_{i s}^{(\mathrm{t})}+\overline{\mathbf{y}}_{i n}^{(\mathrm{t})}=\overline{\boldsymbol{\Phi}}_{i}\left(\overline{\mathbf{w}}_{i c}^{(\mathrm{t})}+\overline{\mathbf{w}}_{i s}^{(\mathrm{t})}\right)+\overline{\mathbf{y}}_{i n}^{(\mathrm{t})},
$$

where the superscript $(\mathrm{t})$ is used to emphasize the range under test, $\overline{\mathbf{w}}_{i c}^{(\mathrm{t})}$ and $\overline{\mathbf{w}}_{i s}^{(\mathrm{t})}$ are, respectively, $M \times 1$ vectorized clutter and signal entries in the angle-Doppler domain. In addition, $\overline{\mathbf{y}}_{i n}^{(\mathrm{t})}$ denotes its noise component. On the other hand, the matched filter output of received signals in the $n_{l}$ th secondary sample, where the described target signals are absent, is expressed for the $i$ th bistatic pair as,

$$
\overline{\mathbf{y}}_{i}^{\left(n_{l}\right)}=\overline{\mathbf{y}}_{i c}^{\left(n_{l}\right)}+\overline{\mathbf{y}}_{i n}^{\left(n_{l}\right)} .
$$

To ensure the exclusion of the target in the secondary samples, the range cells corresponding to these samples should be sufficiently separated from the range cell under test by one or multiple guard cells in each side [9]. Note that passive radar systems exploit narrowband signals and thus causing a coarse range cell resolution, implying a low probability for a target to move across multiple range cells within the CPI.

Rewrite the clutter term in Eq. (32) as the product of an overdetermined dictionary matrix $\overline{\boldsymbol{\Phi}}_{i}$ and sparse vector $\overline{\mathbf{w}}_{i c}^{\left(n_{l}\right)}$, we have

$$
\overline{\mathbf{y}}_{i}^{\left(n_{l}\right)}=\overline{\mathbf{\Phi}}_{i} \overline{\mathbf{w}}_{i c}^{\left(n_{l}\right)}+\overline{\mathbf{y}}_{i n}^{\left(n_{l}\right)}
$$

where $\overline{\mathbf{w}}_{i c}^{\left(n_{l}\right)} \in \mathcal{C}^{N_{d} N_{s}}$.

In this paper, we consider a small number of secondary data samples from range cells that are close to that under test, and thus it is well justified that they share the same non-zero clutter support, i.e., their scatterers are located in the same positions in the angle-Doppler domain. However, the exact values of the scatterers generally differ. Note that the common support assumption is much more relaxed and practical compared to the i.i.d. requirement. We only need a small number of secondary samples in this case. The sparse clutter entries $\overline{\mathbf{w}}_{i c}^{\left(n_{l}\right)}$ are separately computed for each bistaic pair.

\section{B. Clutter Support Estimation and Clutter Suppression}

The group SBL algorithm described in Section III can be used to recover the sparse clutter profiles described as an MMV model in Eq. (33) and subsequently acquire the 
common clutter support $\overline{\mathbf{\Phi}}_{i, c s}$, which is a submatrix of $\overline{\mathbf{\Phi}}_{i}$ and acts as a collection of the columns whose corresponding coefficients in $\overline{\mathbf{w}}_{i c}^{\left(n_{l}\right)}$ are non-zero. One way to confine the clutter estimate for the range cell under test is to project the received signal to the clutter profile through the weighted least square (WLS) method, expressed as,

$$
\hat{\mathbf{w}}_{i c}^{(\mathrm{t})}=\left(\overline{\boldsymbol{\Phi}}_{i, c s}^{H} \mathbf{U}_{0} \overline{\boldsymbol{\Phi}}_{i, c s}\right)^{-1} \overline{\boldsymbol{\Phi}}_{i, c s}^{H} \mathbf{U}_{0} \mathbf{y},
$$

where $u_{l}$ is the $l$ th diagonal entry of the diagonal weighted matrix $\mathbf{U}_{0}$ and its value is chosen to be the reciprocal of noise variance. A more reliable solution is to estimate the clutter profile $\mathbf{w}_{i c}^{(\mathrm{t})}$ by solving the following equation based on the SBL algorithm, similar to that depicted in Section III,

$$
\overline{\mathbf{y}}_{i}^{(\mathrm{t})}=\overline{\mathbf{\Phi}}_{i, c s} \mathbf{w}_{i c}^{(\mathrm{t})}+\overline{\mathbf{y}}_{i n}^{(\mathrm{t})} .
$$

The posterior mean, as depicted in Eq. (23), will be used as the estimate of $\mathbf{w}_{i c}^{(\mathrm{t})}$. Note that, when compared with Eq. (33), the measurement matrix $\overline{\boldsymbol{\Phi}}_{i}$ is replaced by $\overline{\boldsymbol{\Phi}}_{i, c s}$ corresponding only to the clutter support and $\mathbf{w}_{i c}^{(\mathrm{t})}$ is a corresponding subvector. The target signal term disappeared in Eq. (35), because it is out of the clutter support. As such, the estimated coefficient vector $\hat{\mathbf{w}}_{i c}^{(\mathrm{t})}$ only involves clutter components, whereas the target signals are excluded.

Once the clutter coefficient vector $\hat{\mathbf{w}}_{i c}^{(\mathrm{t})}$ is estimated, the corresponding $\mathrm{CCM}$ is expressed as $[9,22]$

$\hat{\mathbf{R}}_{\mathbf{z}}^{(i)}=\sum_{m=1}^{M}\left|\hat{\mathbf{w}}_{i c}^{(\mathrm{t})}\left(\nu_{i m}, \phi_{m}\right)\right|^{2} \mathbf{h}\left(\nu_{i m}, \phi_{m}\right) \mathbf{h}^{H}\left(\nu_{i m}, \phi_{m}\right)+\hat{\beta}_{i, 0} \mathbf{I}_{N L}$

where $\hat{\beta}_{i, 0}$ represents the noise power in the $i$ th bistatic pair, which is adaptively estimated from the observation data as described above in Eq. (30).

Based on this result, we acquire the weight vector for the range cell under test in the $i$ th bistatic pair as [9]

$$
\mathbf{u}_{i}=\frac{\left[\hat{\mathbf{R}}_{\mathbf{z}}^{(i)}\right]^{-1} \mathbf{h}_{0}\left(\bar{\nu}_{p}, \phi_{m}\right)}{\mathbf{h}_{0}^{H}\left(\bar{\nu}_{p}, \phi_{m}\right)\left[\hat{\mathbf{R}}_{\mathbf{z}}^{(i)}\right]^{-1} \mathbf{h}_{0}\left(\bar{\nu}_{p}, \phi_{m}\right)},
$$

where $\mathbf{h}_{0}\left(\bar{\nu}_{p}, \phi_{m}\right)$ is the spatio-temporal signature of the hypothesized target corresponding to Doppler frequency $\bar{\nu}_{p}$ and spatial angle $\phi_{m}$.

\section{MOTION PARAMETER ESTIMATION IN THE Multi-STATIC PAssive System}

In the conventional mono-static STAP, the Doppler frequency of a moving target only reflects its radial velocity, whereas its 2-D motion parameters cannot be estimated. In the multi-static passive radar system, on the other hand, the 2-D motion parameters can be estimated due to observations from multiple transmitter-receiver pairs with diverse bistatic aspect angles.

Eq. (10) is rewritten according to the geometry of the multi- static passive system, as depicted in Fig. 1, as

$$
\begin{aligned}
\nu_{i q} & =\nu_{i q}^{(T)}+\nu_{i q}^{(R)} \\
\nu_{i q}^{(T)} & =-\frac{1}{\lambda_{i}} \frac{\left[\mathbf{p}_{\mathrm{T}_{i}}-\mathbf{p}_{q}(0)\right]^{T} \cdot \mathbf{v}_{q}}{\left\|\mathbf{p}_{\mathrm{T}_{i}}-\mathbf{p}_{q}(0)\right\|} \\
& =-\frac{\cos \phi_{T_{i}} v_{x q}+\sin \varphi_{T_{i}} \cos \theta_{T_{i}} v_{y q}}{\lambda_{i}} \\
\nu_{i q}^{(R)} & =-\frac{1}{\lambda_{i}}\left[\frac{\left[\mathbf{p}_{\mathrm{R}}(0)-\mathbf{p}_{q}(0)\right]^{T} \cdot \mathbf{v}_{q}}{\left\|\mathbf{p}_{\mathrm{R}}(0)-\mathbf{p}_{q}(0)\right\|}+\frac{\left[\mathbf{p}_{\mathrm{R}}(0)-\mathbf{p}_{q}(0)\right]^{T} \cdot \mathbf{v}_{R}}{\left\|\mathbf{p}_{\mathrm{R}}(0)-\mathbf{p}_{q}(0)\right\|}\right] \\
& =-\frac{1}{\lambda_{i}}\left[\cos \phi_{r} v_{x q}+\sin \varphi_{r} \cos \theta_{r} v_{y q}+\cos \phi_{r} v_{R}\right]
\end{aligned}
$$

where $\phi_{T_{i}}$ and $\phi_{r}$ are the respective cone angles of the $i$ th transmitter and the receiver, whereas $\theta_{T_{i}}$ and $\theta_{r}, \varphi_{T_{i}}$ and $\varphi_{r}$ are respectively the depression angles and azimuth angles of the $i$ th illuminator and the receiver. In Eq. (38), we divide the entire Doppler frequency into two parts, where $\nu_{i q}^{(T)}$ is induced by the relative motion of the $q$ th moving target with respect to the $i$ th illuminator. Thus, it varies with the illuminator position and, as such, enables the estimation of 2-D motion parameters. The other term, $\nu_{i q}^{(R)}$, is induced by the motions of the target and the receiver platform and is invariant to the illuminators.

In the following two subsections, we respectively consider the motion parameter estimation in the presence of a single and multiple moving targets.

\section{Parameter Estimation in Single Moving Target Case}

We first consider a simple case where only one moving target exists in the spatial angle $\phi_{m}$. In the conventional STAP, the estimated weight vector is first used to whiten the clutter profile and perform matched filtering on the spatio-temporal signature of the hypothesized target, and individual constant false alarm rate (CFAR) detection is then applied. We can then acquire the corresponding Doppler frequency of the moving target in each bistatic pair. From Eq. (38), we obtain

$$
\boldsymbol{\nu}=\mathbf{D v}+\mathbf{e}
$$

with

$$
\mathbf{D}=\left[\begin{array}{cc}
\frac{\cos \phi_{r}+\cos \phi_{T_{1}}}{\lambda_{1}} & \frac{\sin \varphi_{r} \cos \theta_{r}+\sin \varphi_{T_{1}} \cos \theta_{T_{1}}}{\lambda_{1}} \\
\vdots & \vdots \\
\frac{\cos \phi_{r}+\cos \phi_{T_{K}}}{\lambda_{K}} & \frac{\sin \varphi_{r} \cos \theta_{r}+\sin \varphi_{T_{K}} \cos \theta_{T_{K}}}{\lambda_{K}}
\end{array}\right],
$$

where $\boldsymbol{\nu}=\left[\nu_{1}, \cdots, \nu_{K}\right]^{T}, \mathbf{v}=\left[v_{x}, v_{y}\right]^{T}$, and $\mathbf{e}=$ $\left[-\cos \phi_{r} v_{R} / \lambda_{1}, \cdots,-\cos \phi_{r} v_{R} / \lambda_{K}\right]^{T}$. Therefore, the 2-D motion parameters can be obtained as the least square (LS) solution to Eq. (41), expressed as

$$
\mathbf{v}=\left(\mathbf{D}^{H} \mathbf{D}\right)^{-1} \mathbf{D}^{H}(\boldsymbol{\nu}-\mathbf{e}) .
$$

Note that the LS algorithm described in Eq. (43) is effective only when the signal power is sufficiently high to allow target detection in each bistatic pair. It may become difficult, however, to detect the moving target and acquire its corresponding Doppler frequency for each bistatic pair when the signals are 
noisy.

\section{B. Parameter Estimation in Multiple Moving Target Case}

Multiple moving targets may appear in the same spatial angle $\phi_{m}$ and are unresolvable in bistatic ranges due to a coarse range resolution. In this case, the application of the LS algorithm for motion parameter estimation in the presence of multiple targets requires proper association of the Doppler frequencies with the corresponding targets [46]. The complexity of procedure increases exponentially as the number of targets increases. On the other hand, as we discussed earlier for the single-target case, the LS algorithm works only when the Doppler frequency corresponding to each bistatic pair can be reliably estimated. To perform multi-target motion parameter estimation that is robust for noisy signals, a novel joint parameter estimation method based on sparse reconstruction techniques is proposed.

We first acquire the spatio-temporal weight vector of hypothesized target in the $i$ th bistatic pair specified by the Doppler frequency $\bar{\nu}_{p}$ and the spatial angle $\phi_{m}$ as,

$$
\mathbf{u}_{p i}\left(\phi_{m}\right)=\frac{\left[\hat{\mathbf{R}}_{\mathbf{z}}^{(i)}\right]^{-1} \mathbf{h}\left(\bar{\nu}_{p}, \phi_{m}\right)}{\mathbf{h}^{H}\left(\bar{\nu}_{p}, \phi_{m}\right)\left[\hat{\mathbf{R}}_{\mathbf{z}}^{(i)}\right]^{-1} \mathbf{h}\left(\bar{\nu}_{p}, \phi_{m}\right)},
$$

where

$$
\begin{aligned}
& \mathbf{h}\left(\bar{\nu}_{p}, \phi_{m}\right)=\mathbf{b}\left(\bar{\nu}_{p}\right) \otimes \mathbf{a}\left(\phi_{m}\right), \\
& \mathbf{b}\left(\bar{\nu}_{p}\right)=\left[e^{-j 2 \pi \bar{\nu}_{p}(L-1) T / 2}, e^{-j 2 \pi \bar{\nu}_{p}(L-3) T / 2}, \ldots,\right. \\
&\left.e^{j 2 \pi \bar{\nu}_{p}(L-1) T / 2}\right]^{T},
\end{aligned}
$$

for $p \in[1, \cdots, P]$ with $P$ denoting the total number of the hypothesized Doppler frequency points corresponding to the same spatial angle $\phi_{m}$. The STAP output corresponding to the $i$ th bistatic pair can be expressed as,

$$
g_{p i}\left(\phi_{m}\right)=\mathbf{u}_{p i}^{H}\left(\phi_{m}\right) \overline{\mathbf{y}}_{i}^{(\mathrm{t})} .
$$

In the traditional threshold detection technique, the existence of a target is determined for each individual bistatic pair by choosing a proper threshold. For weak signals, however, target detection based on an individual bistatic pair is not reliable. Consider the fact that, although motion-induced Doppler frequencies differ for each bistatic pair due to the different bistatic angles and carrier frequencies, they share the same motion parameters in the velocity domain and thus have the group sparsity. In the proposed algorithm, we project the spatiotemporal signature to the 2-D velocity vector of $\mathbf{v}=\left[v_{x}, v_{y}\right]^{T}$ in the velocity space. We uniformly discretize 2-D velocity parameters $\left[-v_{x \max }, v_{x \max }\right]$ and $\left[-v_{y \max }, v_{y \max }\right]$ to form an $N_{x} \times N_{y}$ grid. Given a certain spatial angle $\phi_{m}$, the spatiotemporal steering vector of the moving target with motion parameters of $v_{x}^{(a)}$ and $v_{y}^{(b)}$ in the $i$ th bistatic pair is denoted as

$$
\mathbf{h}_{i}^{(a, b)}\left(\phi_{m}\right)=\mathbf{b}\left(f_{i s}^{(a, b)}\left(\phi_{m}\right)\right) \otimes \mathbf{a}\left(\phi_{m}\right)
$$

where

$$
\begin{aligned}
f_{i s}^{(a, b)}\left(\phi_{m}\right)=- & \frac{1}{\lambda_{i}}\left[\left(\cos \phi_{T_{i}}+\cos \phi_{m}\right) v_{x}^{(a)}\right. \\
+ & \left.\left(\sin \varphi_{T_{i}} \cos \theta_{T_{i}}+\sin \varphi_{m} \cos \theta_{r}\right) v_{y}^{(b)}\right] \\
& +\frac{\cos \phi_{m} v_{R}}{\lambda_{i}}
\end{aligned}
$$

is the Doppler frequency for $a \in\left[1, \cdots, N_{x}\right]$ and $b \in$ $\left[1, \cdots, N_{y}\right]$. We construct a dictionary matrix $\boldsymbol{\Theta}^{(m i)} \in$ $\mathcal{C}^{P \times N_{x} N_{y}}$ for the $m$ th spatial angle $\phi_{m}$ under test in the $i$ th bistatic pair and express the entry of $\boldsymbol{\Theta}^{(m i)}$ in the $p$ th row and the $l$ th column as,

$$
\theta^{(m i)}(p, l)=\mathbf{u}_{p i}^{H}\left(\phi_{m}\right) \mathbf{h}_{i}^{(a, b)}\left(\phi_{m}\right),
$$

where $l=(a-1) N_{x}+b, p \in[1, \cdots, P]$, and $l \in$ $\left[1, \cdots, N_{x} N_{y}\right]$.

According to Eq. (31) and Eq. (47), we have,

$$
\begin{aligned}
g_{p i}\left(\phi_{m}\right) & =\mathbf{u}_{p i}^{H}\left(\phi_{m}\right) \overline{\mathbf{y}}_{i s}^{(\mathrm{t})}+\bar{n}_{p i}, \\
\bar{n}_{p i} & =\mathbf{u}_{p i}^{H}\left(\phi_{m}\right) \overline{\mathbf{y}}_{i c}^{(\mathrm{t})}+\mathbf{u}_{p i}^{H}\left(\phi_{m}\right) \overline{\mathbf{y}}_{i n}^{(\mathrm{t})} .
\end{aligned}
$$

The role of the STAP weight vector $\mathbf{u}_{p i}\left(\phi_{m}\right)$ based on the estimated CCM in Eq. (36) is to suppress the clutter and enhance the target signals through matched filtering. The terms caused by the clutter signal $\overline{\mathbf{y}}_{i c}^{(\mathrm{t})}$ and the noise signal $\overline{\mathbf{y}}_{i n}^{(\mathrm{t})}$ in the output $g_{p i}\left(\phi_{m}\right)$ can be defined as a new noise signal $\bar{n}_{p i}$ in Eq. (52) in the new model. As a result, the output signal vector $\mathbf{g}_{i}=\left[g_{1 i}, \cdots, g_{P i}\right]^{T} \in \mathcal{C}^{P}$ can be expressed as,

$$
\mathbf{g}_{i}=\boldsymbol{\Theta}^{(m i)} \boldsymbol{\omega}^{(m i)}+\overline{\mathbf{n}}_{i}, \quad i \in[1, \cdots, K]
$$

where $\boldsymbol{\omega}^{(m i)} \in \mathcal{C}^{N_{x} N_{y}}$ and $\overline{\mathbf{n}}_{i}=\left[\bar{n}_{p i}, \cdots, \bar{n}_{P i}\right]^{T}$. The motion parameter estimate problem can thus be formulated as the sparse reconstruction problem of $\boldsymbol{\omega}^{(m i)}$ from $\mathbf{g}_{i}$. It should be pointed out that the vectors $\left\{\boldsymbol{\omega}^{(m i)}\right\}_{i=1}^{K}$ share the same velocity supports across all bistatic pairs because the true motion parameters are shared by all illuminators in the velocity domain, although their corresponding Doppler frequencies in each bistatic pair are different. It is interesting to note that Eq. (53) is a group sparse reconstruction problem. Therefore, it can be solved using the aforementioned group SBL method, and the estimated motion parameters are the posterior mean depicted in Eq. (23). However, consider the fact that the number of moving targets in each range cell is usually small and can be estimated, other CS methods, such as the BOMP algorithm, may also yield good performance with a lower computational complexity, provided that the their velocities are not very close.

\section{Simulation Results}

Consider a multi-static passive radar system including $K=$ 4 stationary illuminators and 1 moving receiver. As depicted in Fig. 2, the illuminators are located several kilometers away from the scene center with a height of $150 \mathrm{~m}$, and their respective aspect angles are $0^{\circ}, 90^{\circ},-150^{\circ}$ and $-45^{\circ}$. These illuminators emit DVB-T signals with their respective carrier frequencies from $800 \mathrm{MHz}$ to $860 \mathrm{MHz}$ with a $20 \mathrm{MHz}$ frequency interval. The initial position of the receiver is $[0$, 


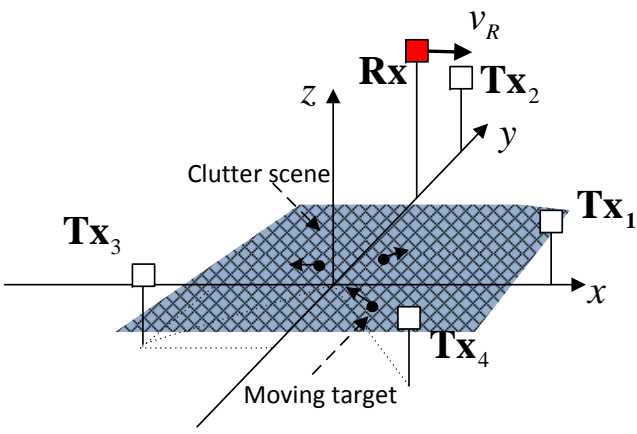

Fig. 2. Geometry of multi-static passive in the simulations.

6 ,8] $\mathrm{km}$, and the receiver is equipped with a 20-element ULA whose inter-element spacing is half of the minimum wavelength. The velocity of the receiver is $v_{\mathrm{R}}=[100,0,0]$ $\mathrm{m} / \mathrm{s}$. The azimuth sampling frequency is $600 \mathrm{~Hz}$, yielding an unaliased observation of Doppler frequency between $-300 \mathrm{~Hz}$ and $300 \mathrm{~Hz} .30$ azimuthal samples are used to perform STAP. As such, a total number of $N L=600$ spatio-temporal samples is adopted.

In the group SBL reconstruction processing, we empirically initialize $\beta_{0}=10^{-4} \times \operatorname{std}(\mathbf{y})^{2}$, the threshold $\eta=10^{-6}$ for stopping the algorithm, and the maximum number of iterations is 5000. As the clutter is usually much stronger than the noise, the above values of parameters $\beta_{0}$ and $\eta$ are suited in the underlying clutter profile reconstruction problem. In this case, a large value of threshold $\eta$ will degrade the reconstruction performance because the algorithm would be terminated before a finer steady state performance could be reached. Compared to $\eta$, the impact of the noise variance $\beta_{0}$ on the reconstruction performance is less direct. A discussion about this impact is available at [51].

\section{A. Clutter Profiles Estimation}

1) Clutter Profiles with Group Sparsity: In Fig. 3, we show the clutter profiles in the 1 st bistatic pair with $n_{t}=4$ nearby secondary samples in the angle-Doppler domain, where each scatterer coefficient is drawn from a complex Gaussian distribution. The angle-Doppler profile of the clutter is discretized into a grid of $N_{d}=90$ Doppler bins from $-300 \mathrm{~Hz}$ to $300 \mathrm{~Hz}$ and $N_{s}=40$ angle bins from $-180^{\circ}$ to $180^{\circ}$, yielding 3600 entries. It is shown in [52] that the value of $N_{s} N_{d}$ should be chosen between $4 N L$ and $16 N L$. In our simulations, the clutter profiles in each angular bin are represented by two adjacent non-zero random complex entries in each bistatic pair, and thus the total number of non-zero entries is 80 , as shown in Fig. 3. It is observed that these clutter profiles in the nearby range cells share the same group sparsity. In addition, a complex Gaussian noise is added and the clutter-to-noise ratio (CNR) is $40 \mathrm{~dB}$.

2) Reconstruction of Clutter Profiles Based on Group SBL $\therefore$ In the following, the group SBL algorithm is compared with several state-of-the-art group sparse reconstruction algorithms, including BOMP [40], GLasso [41], and M-FOCUSS [43]. To quantitatively evaluate the performances of those algorithms, we introduce the normalized mean square error (NMSE), defined as $\left\|\hat{\boldsymbol{\vartheta}}-\boldsymbol{\vartheta}_{\text {gen }}\right\|^{2} /\left\|\boldsymbol{\vartheta}_{\text {gen }}\right\|^{2}$, to be the performance metric, where $\hat{\vartheta}$ is the estimate of the scatterer coefficient vector $\boldsymbol{\vartheta}_{\text {gen }}$.

In this simulation, one neighboring secondary sample in each bistatic pair is used to estimate the common clutter support. Fig. 4(a) shows the original clutter profile support in the 1st bistatic pair, whereas Figs. 4(b)-4(f) shows the estimated support from different algorithms, where the BOMP algorithm assumes the knowledge of the true sparsity. The BOMP fails to recover the clutter profile and leads to a large number of spurious clutter entries around the true positions because of the high coherence in the measurement matrix. Fig. 5(a) shows the NMSE of the reconstructed clutter profile to quantitatively compare the performance of the different methods in reconstructing the clutter profile in the nearby range cells. It is observed that the M-FOCUSS algorithm offers a lower reconstruction error than that by GLasso, and the group SBL method yields the least spurious entries with a minimum NMSE. These results clearly demonstrate the superiority of the group SBL method over other methods in the presence of highly coherent measurement matrix.

To demonstrate the advantage of group sparsity, we separately perform the SBL algorithm and acquire individual clutter support in each range cell. In the latter case, the overall clutter support is obtained as the union of the individual clutter supports corresponding to each range cell. This technique is referred to as the single SBL (S-SBL). It is shown in Fig. 4(f) that, without taking the advantage of the group sparsity between multiple range cells, the S-SBL yields a poor clutter support estimation.

Once the clutter support is estimated, the clutter profiles in the range cell under test can be acquired by using the WLS algorithm in Eq. (34) and the CS algorithms in Eq. (35). The corresponding NMSEs are respectively shown in Figs. 5(b) and 5(c). It is observed that the reconstruction error obtained from the WLS algorithm, shown in Fig. 5(b), is generally higher than that obtained from the corresponding sparse reconstruction method as shown in Fig. 5(c). Due to superiority of the CS method over the WLS method, we use the CS method for the processing in the sequel.

Fig. 6(a) shows the clutter profile of the range cell under test in the 1st bistatic pair. By using the method proposed in Section IV, we estimate the clutter coefficients based on the clutter supports which are respectively obtained from the BOMP, GLasso, M-FOCUSS, S-SBL, and group SBL algorithms. The results are respectively depicted in Figs. 6(b)6(f). To quantitatively evaluate the performance of clutter scatterer coefficients, the output signal-to-inference-plus-noise ratio (SINR) loss, which is defined as the difference between output SINR and the output signal-to-noise ratio (SNR) [9], is evaluated, and the results are depicted in Fig. 5(d). It is observed that the SINR loss of the group SBL algorithm closely approaches the Clairvoyant solution. This is attributed to the group SBL algorithm which, under the MMV model based on nonparametric Bayesian model, can acquire an improved clutter profile estimation, even though the measurement matrix is highly coherent.

To summarize, the proposed method avoids the restrictive 

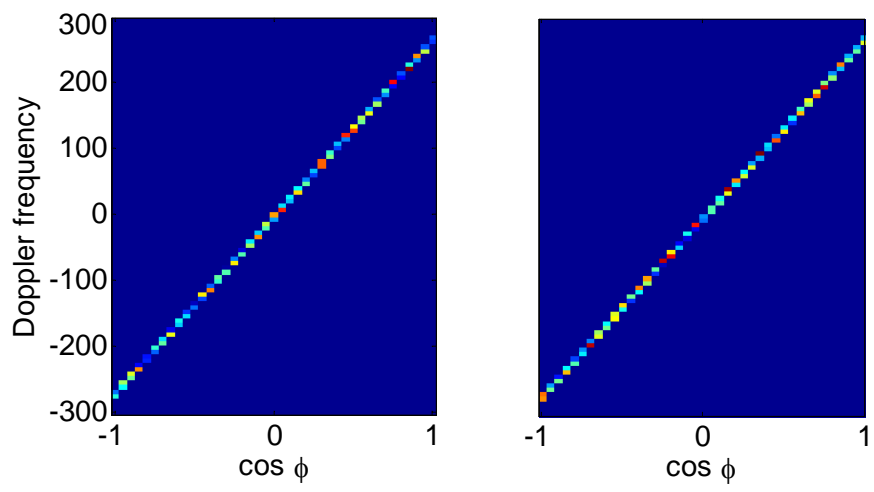

Fig. 3. Clutter profiles with 4 nearby secondary samples.

requirement of a high number of the secondary data samples as in the conventional STAP and acquires an accurate estimation of the CCM for clutter suppression by utilizing only a small number of secondary samples.

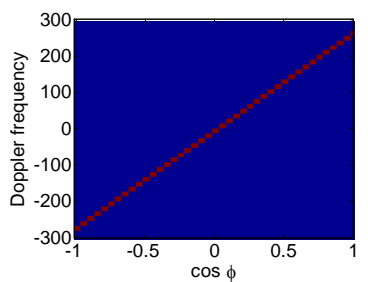

(a)

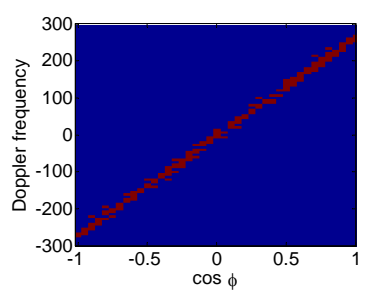

(c)

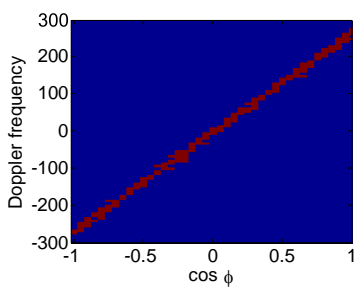

(e)

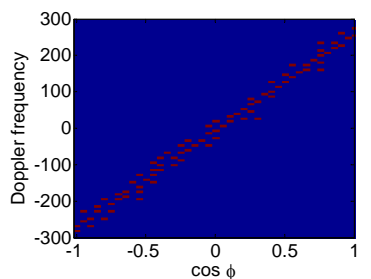

(b)

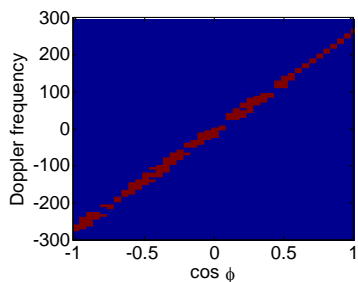

(d)

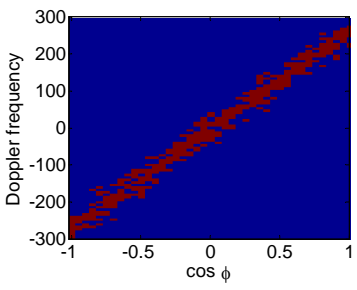

(f)
Fig. 4. Estimated clutter profile support in the 1st bistatic pair and estimated NMSE. (a) Original clutter support. (b) Reconstructed clutter support using BOMP. (c) Reconstructed clutter support using GLasso. (d) Reconstructed clutter support using M-FOCUSS. (e) Reconstructed clutter support using group SBL. (f) Reconstructed clutter support using S-SBL.

3) Effect of Clutter Correlation on Reconstructed Clutter Profile: All simulations above are performed under the assumption that the clutter scattering coefficients in nearby range cells and range cell under test are independent. In practice, this assumption may not be met in the real-world clutter environment. In this case, we investigate effect of the correlated clutter on the sparse clutter reconstruction in
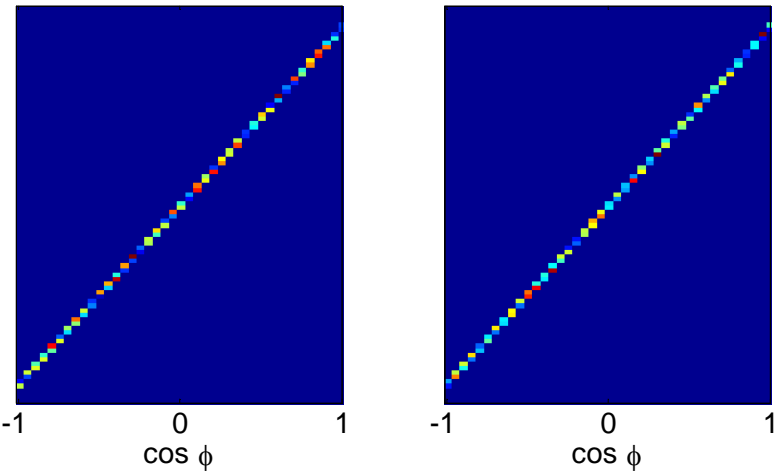

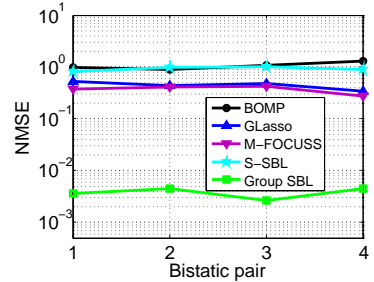

(a)

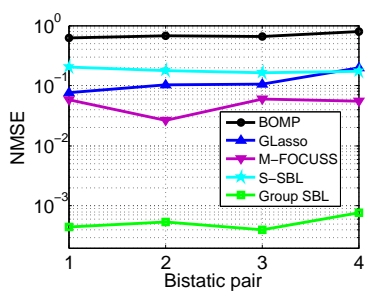

(c)

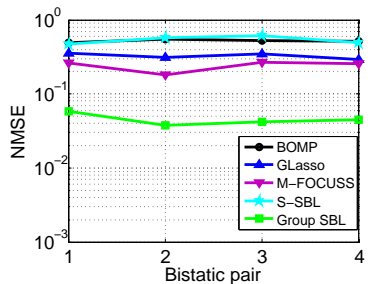

(b)

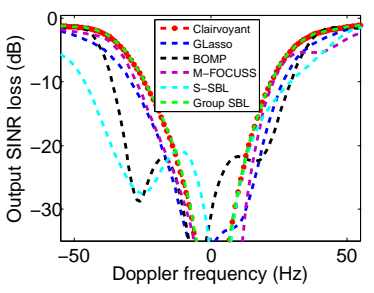

(d)
Fig. 5. Clutter reconstruction and output SINR loss Performances. (a) NMSE of the reconstructed clutter profiles in the nearby range cells obtained from different methods. (b) NMSE of the estimated clutter profile in the range cells under test using the WLS algorithm. Each curve corresponds to a different clutter support estimation method. (c) NMSE of the reconstructed clutter profiles in the range cells under test using the CS algorithm. Each curve corresponds to a different clutter support estimation method. (d) Output SINR loss corresponding to Fig. 5(c).

the proposed method. Fig. 7 shows the reconstructed average NMSE versus correlation of the clutter scattering coefficients, which varies from 0 to 0.9 . It is observed that the proposed method has outstanding reconstruction performance with a very small NMSE, no matter what the correlation is. As a result, we can conclude that the proposed method is robust to the clutter correlation.

\section{B. Motion Parameter Estimation}

1) Parameter Estimation in Single Moving Target Case: In the following simulation, a moving target located at spatial angle $\phi_{m}=106.86^{\circ}$, with $x$ - and $y$-axis velocities of $(15.0,10.2) \mathrm{m} / \mathrm{s}$, is added in the under test range cell. The Doppler frequencies corresponding to the four bistatic pairs are respectively $-131.76 \mathrm{~Hz},-121.93 \mathrm{~Hz},-45.70 \mathrm{~Hz}$, and $-108.37 \mathrm{~Hz}$. These Doppler frequencies are shown in vertical 


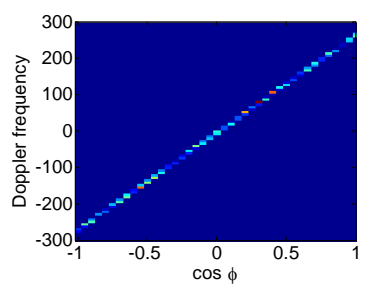

(a)

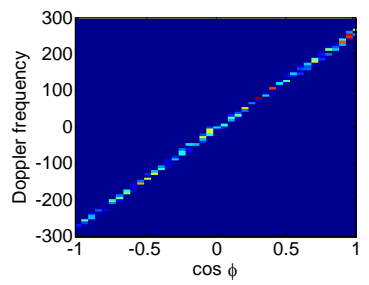

(c)

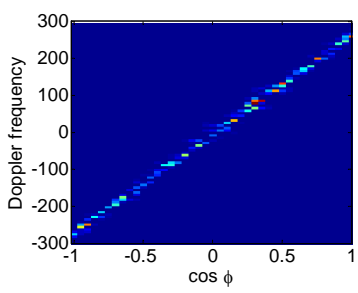

(e)

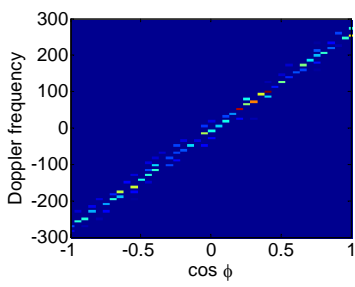

(b)

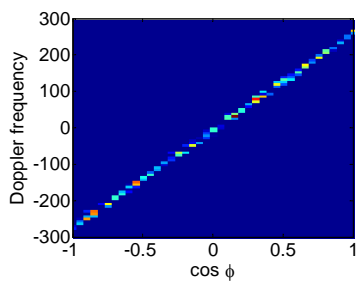

(d)

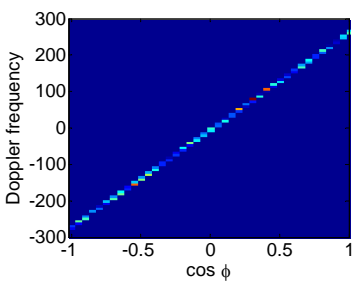

(f)
Fig. 6. Estimated clutter profile in the range cells under test in the 1 st bistatic pair. (a) Original clutter profile. (b) Estimated clutter profile using BOMP. (c) Estimated clutter profile using GLasso. (d) Estimated clutter profile using M-FOCUSS. (e) Estimated clutter profile using S-SBL. (f) Estimated clutter profile using the proposed method.

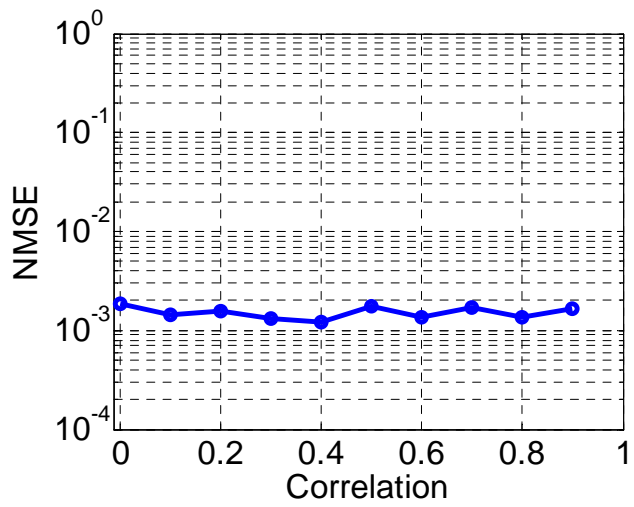

Fig. 7. Effect of clutter correlation on NMSE.

dash lines in Fig. 8(a). The signal-to-clutter ratio (SCR) is -36 dB. Fig. 8(a) shows the output amplitudes of the conventional STAP in which the weight vector is obtained using the group SBL algorithm under the MMV model. In the traditional way, the maximum peaks will be selected. The corresponding estimated frequencies are respectively $-129.20 \mathrm{~Hz},-128.38$ $\mathrm{Hz},-44.51 \mathrm{~Hz}$ and $-115.02 \mathrm{~Hz}$ for the four bistatic pairs through 32 times of interpolation operation. We computer the motion parameters by utilizing the WLS approach described in Eq. (43) and acquire the result of $v_{x}=15.30 \mathrm{~m} / \mathrm{s}$ and $v_{y}=10.12 \mathrm{~m} / \mathrm{s}$. The estimated Doppler frequencies in the 2nd and 4th bistatic pairs have an offset of about $6.45 \mathrm{~Hz}$ and
$6.65 \mathrm{~Hz}$, respectively, yielding velocity estimation errors. The CS-based method proposed in Section V effectively alleviates this effect by utilizing multiple transmitters configuration in the passive radar system. The group SBL is used to estimate the 2-D motion parameters, where the ranges of $v_{x}$ and $v_{y}$ are both from $-50.0 \mathrm{~m} / \mathrm{s}$ to $50.0 \mathrm{~m} / \mathrm{s}$ and the velocity step is 0.5 $\mathrm{m} / \mathrm{s}$. We acquire the estimated motion parameter of $v_{x}=15.0$ $\mathrm{m} / \mathrm{s}$ and $v_{y}=10.0 \mathrm{~m} / \mathrm{s}$.

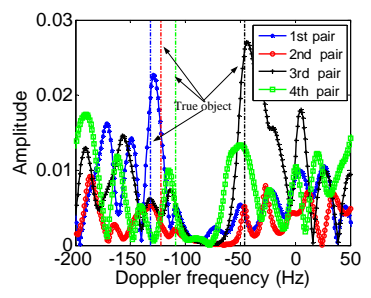

(a)

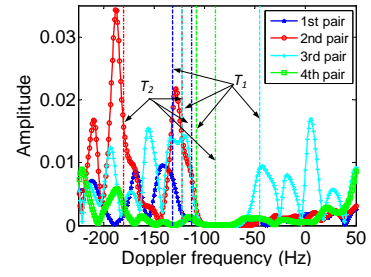

(b)
Fig. 8. Motion parameter estimation of moving targets. (a) Single target; (b) Multiple targets.

2) Parameter Estimation in the Multiple Moving Targets Case: Next, we consider a more general case with multiple moving targets and a low SNR. We add an additional moving target with motion parameters $v_{x}=-5.6 \mathrm{~m} / \mathrm{s}$ and $v_{y}=18.0 \mathrm{~m} / \mathrm{s}$ in the same spatial angle $\phi_{m}=106.86^{\circ}$. All other parameters remain the same as those in the single target case. Fig. 8(b) shows the output amplitudes in each bistatic pair, where $T_{1}$ and $T_{2}$ represent the corresponding Doppler frequencies from the 1st and 2nd moving targets, respectively. The vertical lines respectively denote the true Doppler frequencies of those two targets corresponding to the four bistatic pairs. It is noted that the output amplitude corresponding to the 2nd moving target in the 4th pair, which is located around a true Doppler frequency of $-89.33 \mathrm{~Hz}$, is nearly zero. In this case, the Doppler frequency offset caused by the target motion parameter is only $-6.2 \mathrm{~Hz}$ and, as such, the moving target signal is unresolvable from the clutter and is thus substantially suppressed by the designed spatio-temporal filter. The individual Doppler frequencies are difficult to be accurately estimated in this case and, as such, the LS algorithm completely fails to determine the motion parameters.

Before we present the results obtained from the group SBL algorithm, we perform a conventional non-coherent image fusion method to estimate motion parameters of multiple targets [46]. Fig. 8(b) shows the output amplitude distributions with respect to the Doppler frequency in each bistatic pair. Based on the relationship in Eq. (49) between the Doppler frequencies and motion parameters, we represent those amplitude distributions in the $v_{x}$ and $v_{y}$ domain, as shown in Figs. 9(a)-9(d). One Doppler frequency corresponds to a line in the velocity domain according to the linear relationship in Eq. (49) and the strength of the line is represented by the output amplitude in each Doppler frequency point. The slope of the line is completely determined by the geometry of the illuminators and the receiver, and thus differs for each bistatic pair. For comparison, we depict a heat image in Fig. 9(e) as the result of an intuitional non-coherent method that exploits those acquired images in each bistatic pair. However, 
this method has poor velocity resolution. As can be observed, the combined strength formed from a relatively weak target may still be weaker than the sidelobes of a strong target due to the difference in the target signal power. Accordingly, it is difficult to estimate the correct motion parameters. Fig. 9(f) shows the result obtained from the proposed method. It is clear that the proposed algorithm successfully estimates the motion parameters with $[15.0,10.5] \mathrm{m} / \mathrm{s}$ for the 1 st moving target and $[-5.5,18.5] \mathrm{m} / \mathrm{s}$ for the 2 nd moving target.

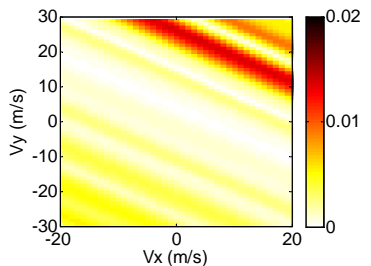

(a)

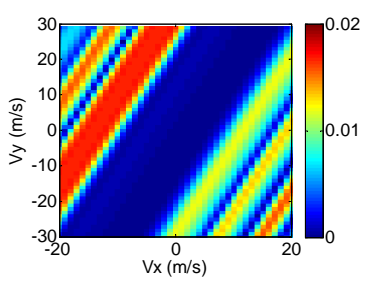

(c)

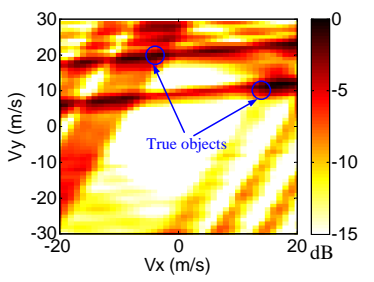

(e)

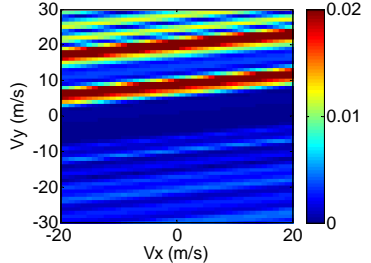

(b)

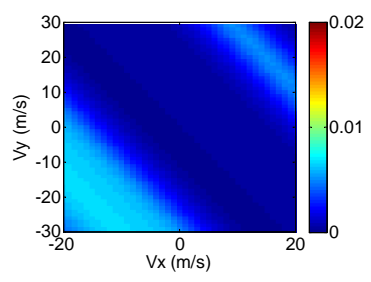

(d)

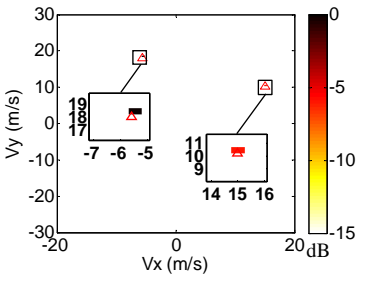

(f)
Fig. 9. Motion parameter estimation in the multiple moving target case. (a)-(d) Outputs amplitude distribution in the 4 bistatic pairs. (e) Motion parameter estimation based on non-coherent fusion. (f) Motion parameter estimation based on the proposed method.

\section{CONCLUSION}

Conventional space-time adaptive processing (STAP) suffers from the high number requirement of independent identically distributed (i.i.d.) secondary data samples for a reliable estimation of statistical clutter covariance matrix (CCM) so as to achieve effective clutter suppression in the range cell under test. One possible solution to relax this condition is to utilize the range cell data under test to estimate its CCM. The sparse Bayesian learning (SBL) technique provides a feasible means for accurate reconstruction of the clutter profile in the angleDoppler domain, particularly when the measurement matrix has a high coherence.

In this paper, a novel method was proposed to estimate the CCM based on a small number of secondary samples. The common clutter support was estimated by exploiting the fact that this support is shared across neighboring range cells. This estimate is used in the range cell under test to acquire the clutter profile with the target signal excluded. This strategy does not require i.i.d. secondary samples, neither does it include the target signals in the estimated CCM. The group sparse problem is effectively solved using the group SBL algorithm in the multiple measurement vector model to achieve accurate and robust performance in the presence of highly coherent measurement matrix that relates the spatiotemporal measurements and the angle-Doppler domain clutter profile.

Another important contribution of this paper was the group sparsity-based two-dimensional motion parameter estimation of a single or multiple ground moving targets. While the traditional least-square solutions provide a simple approach to achieve this objective, they are infeasible when dealing with multiple target case, or when the Doppler estimation from each individual bistatic pair is unreliable. The proposed technique, on the other hand, achieves improved motion parameter estimation in such challenging situations.

\section{REFERENCES}

[1] H. D. Griffths and C. J. Baker, "Passive coherent location radar systems. part 1: Performance prediction," IEE Proc. Radar, Sonar and Navig., vol. 152, no. 3, pp. 153-159, 2005.

[2] N. J. Willis and H. D. Griffiths (eds.), Advances in Bistatic Radar. SciTech, 2007.

[3] S. Barbarossa and A. Farina, "Detection and imaging of moving objects with synthetic aperture radar. part 2: Joint timefrequency analysis by wigner-ville distribution," IEE Proc. Pt. $F$, vol. 139, no. 1, pp. 89-97, 1992.

[4] G. Li, J. Xu, Y. Peng, and X. Xia, "Bistatic linear array SAR for moving target detection, location and imaging with two passive airborne radars," IEEE Trans. Geosci. Remote Sens., vol. 45, no. 3, pp. 554-565, March 2007.

[5] S. Subedi, Y. D. Zhang, M. G. Amin, and B. Himed, "Robust motion parameter estimation in multisatic passive radar," in Proc. European Signal Processing Conf., (Marrakech, Morocco), Sept. 2013.

[6] I. G. Cumming and F. H. Wong, Digital Processing of Synthetic Aperture Radar Data: Algorithms and Implementation. Artech House, 2005.

[7] Q. Wu, M. Xing, C. Qiu, Z. Bao, and T. S. Yeo, "Motion parameter estimation in the SAR system with low PRF sampling," IEEE Geosci. Remote Sens. Lett., vol. 9, no. 4, pp. 710-714, 2010

[8] Q. Wu, M. Xing, and Z. Bao, "Imaging of moving target of dual-channel MIMO-SAR," Journal of System Engineering and Electronics, vol. 32, no. 5, pp. 921-926, 2010.

[9] J. R. Guerci, Space-Time Adaptive Processing for Radar. Artech House, 2003.

[10] M. C. Wicks, M. Rangaswamy, R. Adve, and T. B. Hale, "Space-time adaptive processing: A knowledge-based perspective for airborne radar," IEEE Signal Proc. Mag., vol. 23, no. 1, pp. 51-65, 2006.

[11] I. Reed, J. Mallett, and L. Brennan, "Rapid convergence rate in adaptive arrays," IEEE Trans. Aerospace Electron. Syst., vol. 10, no. 6, pp. 853-863, 1974.

[12] J. Guerci, J. Goldstein, and I. Reed, "Optimal and adaptive reduced-rank STAP," IEEE Trans. Aerospace Electron. Syst., vol. 36, no. 2, pp. 647-663, 2000.

[13] W. L. Melvin, "A STAP overview," IEEE Aerospace and Electronic Systems Magazine, vol. 19, no. 1, pp. 19-35, 2004.

[14] C. H. Gierull and B. Balaji, "Minimal sample support spacetime adaptive processing with fast subspace techniques," IEE Proc. Radar, Sonar and Navig., vol. 149, no. 5, pp. 209-220, 2002.

[15] B. Kang, V. Monga, and M. Rangaswamy, "Constrained ML estimation of structured covariance matrices with applications in radar STAP," in Proc. IEEE 5th International Workshop on 
Computational Advances in Multi-Sensor Adaptive Processing, (Saint Martin), Dec. 2013.

[16] B. Kang, V. Monga, and M. Rangaswamy, "Estimation of structured covariance matrices for radar STAP under practical constraints," in Proc. IEEE Radar Conf., (Cincinnati, OH), May 2014.

[17] X. Neyt, J. Raout, M. Kubica, V. Kubica, S. Roques, M. Acheroy, and J. G. Verly, "Feasibility of STAP for passive GSM-based radar," in Proc. IEEE Radar Conf., (Veronna, NY), pp. 546-551, Apr. 2006.

[18] J. Raout, X. Neyt, and P. Rischette, "Bistatic STAP using DVBT illuminators of opportunity," in Proc. IET Int. Conf. Radar Systems, (Edinburgh, U.K.), Oct. 2007.

[19] I. W. Selesnick, S. U. Pillai, K. Y. Li, and B. Himed, "Angledoppler processing using sparse regularization," in Proc. IEEE ICASSP, (Dallas, TX), March 2010.

[20] J. T. Parker and L. C. Potter, "A Bayesian perspective on sparse regularization for STAP post-processing," in Proc. IEEE Radar Conf., (Washington DC), May 2010.

[21] K. Sun, H. Zhang, G. Li, H. Meng, and X. Wang, "Airborne radar STAP using sparse recovery of clutter spectrum," http://arxiv.org/pdf/1008.4185v1.

[22] K. Sun, H. Meng, Y. Wang, and X. Wang, "Direct data domain STAP using sparse representation of clutter spectrum," Signal Proc., vol. 91, no. 9, pp. 2222-2236, 2011.

[23] Y. D. Zhang and B. Himed, "Space-time adaptive processing in bistatic passive radar exploiting clutter sparsity," in Proc. IEEE Radar Conf., (Cincinati, OH), May 2014.

[24] J. A. Tropp and A. C. Gilbert, "Signal recovery from partial information via orthogonal matching pursuit," IEEE Trans. Info. Theory, vol. 53, no. 12, pp. 4655-4666, 2007.

[25] R. Tibshirani, "Regression shrinkage and selection via the Lasso,” J. Royal. Statist. Soc., vol. 58, no. 1, pp. 267-288, 1996.

[26] Y. Chi and R. Calderbank, "Coherence-based performance guarantees of orthogonal matching pursuit," in Proc. Annual Allerton Conference on Communication, Control, and Computing, (Monticello, IL), 2012.

[27] G. Tang, B. N. Bhaskar, and B. Recht, "Compressed sensing off the grid," IEEE Trans. Info. Theory, vol. 59, no. 11, pp. 74657490, 2013.

[28] E. J. Candes and Y. Plan, "A probabilistic and RIPless theory of compressed sensing," IEEE Trans. Info. Theory, vol. 57, no. 11, pp. 7235-7254, 2011.

[29] M. E. Tipping, "Sparse Bayesian shrinkage and selection learning and the relevance vector machine," J. Machine Learning Research, vol. 1, no. 9, pp. 211-244, 2001.

[30] D. P. Wipf, "Sparse estimation with structured dictionaries," in Proc. Adv. Neural Info. Proc. Syst., (Grendada, Spain), 2011.

[31] M. Carlin, P. Rocca, G. Oliveri, F. Viani, and A. Massa, "Directions-of-arrival estimation through Bayesian compressive sensing strategies," IEEE Trans. Antennas and Propagation, vol. 61, no. 7, pp. 3828-3838, 2013.

[32] S. Qin, Y. D. Zhang, Q. Wu, and M. G. Amin, "DOA estimation of nonparametric spreading spatial spectrum based on Bayesian compressive sensing exploiting intra-task dependency," in Proc. IEEE ICASSP, (Brisbane, Australia), April 2015.

[33] G. Oliveri, P. Rocca, and A. Massa, "A Bayesian compressive sampling-based inversion for imaging sparse scatterers," IEEE Trans. Geosci. Remote Sens., vol. 49, no. 10, pp. 3993-4006, 2013.

[34] L. Poli, G. Oliveri, F. Viani, and A. Massa, "MT-BCS-based microwave imaging approach through minimum-norm current expansion," IEEE Trans. Antennas and Propagation, vol. 61, no. 9 , pp. 4722-4732, Sept. 2013.

[35] Q. Wu, Y. D. Zhang, M. G. Amin, and B. Himed, "Multistatic passive radar SAR imaging based on Bayesian compressive sensing," in Proc. SPIE Defense, Security, and Sensing, (Baltimore, MD), May 2014.

[36] G. Oliveri, M. Carlin, and A. Massa, "Complex-weight sparse linear array synthesis by Bayesian compressive sampling," IEEE Trans. Antennas and Propagation, vol. 60, no. 5, pp. 23092326, May 2012.

[37] G. Oliveri, P. Rocca, and A. Massa, "Reliable diagnosis of large linear arrays-A Bayesian compressive sensing approach," IEEE Trans. Antennas and Propagation, vol. 60, no. 10, pp. 46274636, Oct. 2012.

[38] Q. Wu, Y. D. Zhang, M. G. Amin, and B. Himed, "Complex multitask Bayesian compressive sensing," in Proc. IEEE ICASSP, (Florence, Italy), May 2014.

[39] D. Wipf and S. Nagarajan, "Beamforming using the relevance vector machine," in Proc. Int. Conf. on Machine Learning, pp. 1023-1030, 2007.

[40] J. Huang and T. Zhang, "The benefit of group sparsity," Ann. Statist., vol. 38, no. 4, pp. 1978-2004, 2010.

[41] M. Yuan and Y. Lin, "Model selection and estimation in regression with grouped variables," Journal of the Royal Statistical Society Series B, vol. 68, no. 1, pp. 49-67, 2006.

[42] S. Ji, D. Dunson, and L. Carin, "Multi-task compressive sampling," IEEE Trans. Signal Proc., vol. 57, no. 1, pp. 92-106, 2009.

[43] S. F. Cotter, B. D. Rao, K. Engan, and K. Kreutz-Delgad, "Sparse solutions to linear inverse problems with multiple measurement vectors," IEEE Trans. Signal Proc., vol. 53, no. 7, pp. 2477-2488, 2005.

[44] I. Stojanovic, M. Cetin, and W. C. Karl, "Joint space aspect reconstruction of wide-angle SAR exploiting sparsity," in Proc. SPIE Defense and Security Symposium, (Prague, Czech Republic), May 2008.

[45] N. Ramakrishnan, E. Ertin, and R. L. Moses, "Enhancement of coupled multichannel images using sparsity constraints," IEEE Trans. Image Proc., vol. 19, no. 8, pp. 2115-2126, 2010.

[46] Y. D. Zhang and B. Himed, "Moving target parameter estimation and SFN ghost rejection in multistatic passive radar," in Proc. IEEE Radar Conf., (Ottawa, Canada), April 2013.

[47] S. Subedi, Y. D. Zhang, M. G. Amin, and B. Himed, "Motion parameter estimation of multiple targets in multistatic radar through sparse signal recovery," in Proc. IEEE ICASSP, (Florence, Italy), May 2014.

[48] C. R. Berger, B. Demissie, J. Heckenbach, P. Willett, and S. Zhou, "Signal processing for passive radar using OFDM waveforms," IEEE J. Selected Topics in Signal Proc., vol. 4, no. 1, pp. 226-238, 2010.

[49] D. J. C. MacKay, "Bayesian interpolation," Neural Comput., vol. 4, no. 3, pp. 415-447, 1992.

[50] M. E. Tipping and A. C. Faul, "Fast marginal likelihood maximisation for sparse Bayesian models," in Proc. of the Ninth International Workshop on Artificial Intelligence and Statistics, (Key West, FL), 2003.

[51] Z. Zhang and B. D. Rao, "Clarify some issues on the sparse Bayesian learning for sparse signal recovery," tech. rep., http: //dsp.ucsd.edu/ zhilin/papers/clarify.pdf.

[52] Z. C. Yang, Z. Liu, X. Li, and L. Nie, "Performance analysis of STAP algorithms based on fast sparse recovery techniques," Progress In Electromagnetics Research B, vol. 41, no. 2, pp. 251-268, 2012. 